\title{
David Chapman
}

University of East London.

University of East London

Docklands Campus

4 -16 University Way

London E16 2RD

Email: d.m.chapman@uel.ac.uk

\section{Context-Based Sound and the Ecological Theory of Perception}

\begin{abstract}
This paper aims to investigate the ways in which context-based sonic art is capable of furthering a knowledge and understanding of place based on the initial perceptual encounter. How might this perceptual encounter operate in terms of a sound work's affective dimension? To explore these issues I draw upon James J. Gibson's ecological theory of perception and Gernot Böhme's concept of an 'aesthetic of atmospheres'.

Within the ecological model of perception an individual can be regarded as a 'perceptual system': a mobile organism that seeks information from a coherent environment. I relate this concept to notions of the spatial address of environmental sound work in order to explore (a) how the human perceptual apparatus relates to the sonic environment in its mediated form and (b) how this impacts on individuals' ability to experience such work as complex sonic 'environments'. Can the ecological theory of perception aid the understanding of how the listener engages with context-based work? In proposing answers to this question, this paper advances a coherent analytical framework that may lead us to a more systematic grasp of the ways in which individuals engage aesthetically with sonic space and environment. I illustrate this methodology through an examination of some of the recorded work of sound artist Chris Watson.
\end{abstract}

\section{INTRODUCTION}

The notion of context-based sound work ${ }^{\mathrm{i}}$ opens up a particular set of issues and potentials. The 'context' suggests that a sound work, both in its production and / or presentation, has to be understood as a response to the complex interactions that constitute 'place'. That is to say, place not just as a geographical location but as the site of lived experience, multiple histories, cultural and biological diversity and political contestation. The idea of place is itself a shifting category, its specificity 
under pressure by a homogenising economic and political culture that operates to flatten out its distinctiveness. To render place, to use the terminology of Marc Augé, as 'non-place': 'a space which cannot be defined as relational, or historical, or concerned with identity' (Augé 1995: 92). ${ }^{\text {ii }}$ This engagement with cultural and social spaces feeds a wide-ranging debate on the politics of 'context-based' work, as indicated by the variety of terms used to describe and delineate work that might fall into this category. ${ }^{\text {iii }}$ Context-based work is also constantly having to reconfigure its modes of address in response to new forms of art practices and emerging technologies, let alone the shifting policy and economic agendas which often prefigure the generation of work in the first instance.

Given these issues, work that clearly addresses the specifics of context is mainly discussed as operating on a symbolic level, or regarded as resting on an indexical relationship between place and the sound elements it contains and generates. However, the central question I want to examine here is how might an understanding of context-based work be derived from the initial perceptual encounter? How might context be understood at the level of perception, and the transfer of contextual knowledge from work to listener, without recourse to spoken language? Furthermore, what are the parameters of this experience? Can it be conveyed in documentation media which separates the sonic experience from its place of recording?

In installation practice, context-based sound works are in general rarely presented or experienced as merely sound. More often than not sound installations are presented and experienced in relation to sculptural forms or visual media, and may reference a range of topographical, technological and architectural elements, alongside cultural / personal histories linked to its site of generation and / or exhibition. ${ }^{\text {iv }}$ But how are sound-based works considered in relation to a broader ecology of experience? In reconsidering 'sound as sound' and rebalancing the aural in respect to the dominance of ocularcentric cultural practice and criticism, it is important this is not just replaced by an 'auricularcentric' position, where 'listening' over-rides the consideration of all the other senses. From this perspective the notion of the soundscape itself is clearly open to critique. Anthropologist Tim Ingold (2007) challenges its usefulness as a model for considering sound in the environment and rejects the breaking up of the landscape through a process of 'scaping' - a process which 'slices-up' the sensory apparatus into discrete registers by which we may experience an environment. As he states, '(i)n ordinary perceptual practices these registers co-operate so closely, and with such overlap of function, that their respective contributions are impossible to tease apart' (Ingold 2007: 10). The idea is of the 
perceptual faculties co-operating as a 'system', with the distinction between the different senses in play being hard to differentiate. Sound, if central to the specificity of these installations, should not be divorced from these other elements; even though sound has a particular function as to how such elements might be articulated and understood. If, therefore, we are to consider 'all the senses', how might this approach translate into a mode of analysis capable of accounting for the totality of the experience presented by context-based work? Further, what might this mode of analysis have to offer producers of context-based sound work?

To explore these issues I will draw upon James Gibson's ecological theory of perception and Gernot Böhme's concept of an 'aesthetic of atmospheres'. I argue that these two approaches provide a basis for understanding how perceptual processes function in relation to environmental information, as well as how this information has an affective or emotional force. Using these theoretical perspectives, I explore ways in which we might understand how audiences engage with work based on environmental recordings and producers consider their production strategies. In order to provide an example of how the methodology proposed here might function in practice, I will apply it to some of the recordings produced by sound artist Chris Watson.

\section{THE ECOLOGICAL PERCEPTION OF SOUND}

First of all we must approach context-based installation and soundscape composition as media objects which have been created through a number of production and post-production processes. Chris Watson made the following observation in an interview with David Toop:

On location, to perceive what I may later regard to be memorable sounds, there are two significant characteristics: 1 Clarity $\& 2$ Depth. Clarity being not coloured by other irrelevant sounds or interruptions. Depth being the ability to follow the sound, or its reverberation, into the distance. To be able to listen to the full envelope of the sound. Of course the paradox is that some sounds can convey clarity and depth even when played back or broadcast over the compressed mediums you refer to (television, radio, computers etc.). I'm fascinated by this but can't explain it! (Watson in Toop 2004: 51)

Although Watson is talking primarily about field recordings of natural environments, I would argue that the phenomenon he describes and his observations about it beg a 
whole series of questions on the general sonic environment, the technological processes of sound recording and diffusion, and the core issue of human perception. ${ }^{\mathrm{v}} \mathrm{I}$ want to argue that a methodology is required that can embrace all these aspects and provide a basis for exploring and explaining the phenomenon described by Watson: the ability of the perceptual apparatus to probe and explore sonic space even through the degradation of signal that occurs through various forms of transmission. That is to say, in spite of this mediation, and thus translation into another space, something of the original informational structure of sound operating within and contoured by a particular place can still be conveyed and understood. That sound, even when lacking any other forms of sensory information linked to a particular environment, is powerfully evocative of the experience of place.

Before looking at Gibson's perspectives in more detail, it is useful to open up some wider issues in relation to the critical approach to sound art. Christoph Cox (2011) poses the question: why does sound art remain so undertheorised? Cox's answer, from the perspective of contemporary cultural theory, is that the prevailing theoretical models (that is to say approaches based on semiotics, psychoanalysis, poststructuralism and deconstruction) are inadequate to the task because they have been developed to deal with textual and visual forms of culture. As he puts it:

Cultural criticism and theory is taken to be an interpretive enterprise that consists in tracking signs or representations (images, texts, symptoms, etc) through the associative networks that give them meaning, networks that are always in flux, thus insuring that meaning is never stable. Rejecting realism, which would claim direct access to reality, contemporary cultural theory and criticism tends to maintain that experience is always mediated by the symbolic field. (Cox 2011: 146)

Although for Cox these theoretical approaches have been undeniably productive, the insistence on the dominance and privileging of human symbolic systems and association brings in to play an anthropocentric tendency which aligns it with many of the metaphysical and theological positions which hold that humans and their attributes are above nature. If these are positions that critical theory specifically seeks to challenge, they may (and often do) have the effect of allowing in by the 'backdoor' various forms of essentialism. As Cox writes,

theories of textuality or discursivity implicitly support a separation between culture (the domain of signification, representation, and meaning) and nature (the domain of 
inert, dumb matter). Nature is either cast aside as in-significant or deemed a cultural projection, a social construction. (Cox 2011: 147)

For Cox this problem can be addressed not by developing a sound specific theory but by shifting to a materialist / realist account of sound to enable a more theoretically robust framework for the analysis of both sound arts and art in general.

There is a definite turn to a realist epistemology in the methodology I propose here, based as it is on James J. Gibson's $(1966,1979$, 1982) ecological theory of perception. Although this model does not make a claim to a full blown materialism Gibson's realism extending only to those elements open to perception - it nevertheless posits the environment as a coherent informational matrix, the coherence of which does neither rely on a mental construction nor on the perceiver's symbolic interpretation. Gibson also clearly shifts the emphasis away from a privileging of the human. As the ecological model makes clear, its purpose is to describe processes that operate for animals more generally, focussing on evolutionary processes and survival imperatives relating to an organism's successful functioning in the environment. ${ }^{\mathrm{vi}}$

The work of James Gibson has been of growing notice to musicians and sound artists, particularly his concept of 'affordance'. vii This is in part because of the work of musicologist and psychologist Eric Clarke's (2005) who draws on the ecological perceptual theory of James Gibson and applies it to music, specifically classical and rock. However, here I want to consider the productivity of this approach for an investigation of broader categories of sonic art and in particular work based on environmental recordings. If Gibson is known primarily for his work on visual perception, his earlier work also dealt to a certain extent with the aural. Most importantly for my argument here, Gibson viewed perception as an operation of the brain and the senses as a 'perceptual system' functioning in a mobile body (Gibson 1966). The mobility of the body is of course highly pertinent to site-specific and installation work: such work generally encourages the audience to move through the space rather than to adopt static viewing or listening positions. So, while recognising that Clarke's study concentrates on more conventional definitions of music, the general principles of his conceptualisation can be maintained and might be applied mutatis mutandis to the specifics of sound art as a distinct category of practice.

To look briefly at the way Clarke applies ecological theory, one can say his main objective is a discussion of the different ways the listener interacts with music as part of a general auditory environment. He discusses listening to music as 'the 
continuous awareness of meaning', and considers 'musical materials in relation to perceptual capacities'. In this way Clarke attempts to steer an understanding of musical meaning away from the customary linguistic or semiotic approaches, towards a method based more on questions of perception. He differentiates between gaining an understanding of music through the act, and at the point of listening, and reflecting or thinking about music when not actually engaged in listening to it. As he states, ' $[u]$ nder those circumstances music is imagined or recalled, rather than perceived, as nothing is going on in the peripheral auditory system' (Clarke 2005: 5). Therefore, the emphasis is on deriving meaning from music on the basis of the information that is engrained in the sounds themselves.

A key point in differentiating the ecological approach from other psychological models is the emphasis placed on the structure of information in the environment that the perceptual system 'picks up'. This idea of a 'structure' of information in the environment differs from cognitive, 'information-processing' type approaches that broadly take the environment to be a mass of complex and confusing information that the individual organises and structures as an internalised model. While it should be noted that some contemporary forms of the information processing model are much more integrative of the dynamic role of the perception of environmental data in

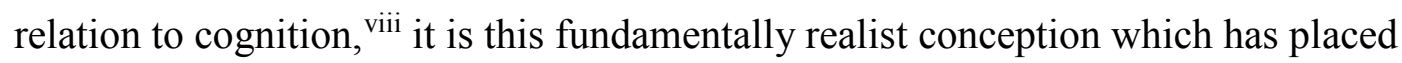
Gibson's work outside, or against, what might be considered the 'mainstream' of psychological models of perception. Ecological perception leads to a focus on what may be 'directly specified by environmental information - not what a perceiving organism can interpret in, or construct from, a stimulus' (Clarke 2005: 17-18). To reinforce this point, Clarke refers explicitly to Gibson:

Instead of supposing that the brain constructs or computes the objective information from a kaleidoscopic inflow of sensations, we may suppose that the orientating of the organs of perception is governed by the brain so that the whole system of input and output resonates to the external information. (Gibson in Clarke 2005: 18)

The processes of perception can be seen as developing an 'attunement' to the environment because '[p]erception is a self-tuning process, in which the pick-up of environmental information is intrinsically reinforcing, so that the system self-adjusts so as to optimise its resonance with the environment' (Clarke 2005: 19). Or as Gibson summarises this in more straightforward terms, a 'system hunts until it achieves clarity' (Gibson 1966: 171). 
Taking forward the basic idea that perception occurs in the mobile body, perception and action can be seen as interdependent. Therefore, we should regard perception as 'essentially exploratory, seeking out sources of stimulation in order to discover more about the environment' (Clarke 2005: 19, my emphasis) - for example, leaning forward to touch or turning the head to see better something glimpsed in the peripheral vision and so on. However, many aesthetic and entertainment experiences are structured to prevent, or at least, curtail these 'perception-action' functions, for instance the way the viewer or listener is prevented from touching a painting or video screen in a gallery, or the enforced codes of behaviour at classical concerts (remaining seated, regulated applause at prescribed moments and so forth). This 'curtailment' is particularly noticeable in 'high' art forms in Western countries - in music a whole series of disciplines and etiquettes developed in the $19^{\text {th }}$ century in order to move the listening of music towards a more contemplative mode (Crary 1999; Clarke 2005). What might be regarded as a form of 'perceptual management'. This can be broadly opposed to folk and pop culture and some later avant-garde art practices, which overtly encourage a greater degree of active participation

This idea of perception as 'exploratory' emphasises the notion of the perceptual system working within a mobile body but might we also imagine that perception projects outwards from the body: that it probes the structure of the information in the environment, as opposed to a being a more 'passive' receptor of incoming date? If we accept this last point, this exploratory facility, if enhanced through mobility, also works for the 'static' listener.

If the perceptual system picks up information from the environment in the first instance, it is also important to consider what is the nature of this information in terms of a more complex engagement with the environment. That is to say, what qualitative or affective dimension might also be discernable?

\section{AN AESTHETICS OF ATMOSPHERES}

If we use Gibson's ecological theory to gain a better understanding of the perceptual processes, how does this allow sonic work to communicate something about space and place? In a paper on music and acoustic ecology, the German philosopher Gernot Böhme (2000) proposes an 'Aesthetics of Atmospheres' which emerges out of a more general notion of an 'Ecological Nature Aesthetics': the exploration of the relationship between the quality of the environment and human sensibilities. The development of Ecological Nature Aesthetics is an attempt to move an understanding 
of ecology beyond the preserve of the natural sciences or, in more popular discourse, as a sphere that has fallen into crisis and is synonymous with issues of man-made environmental degradation. The intention is to develop ecology into a fully-fledged science of human environments, which also considers qualities in the environment that might be experienced 'aesthetically'. For Böhme the notion of atmospheres became the primary focus of this broader aesthetic. As he outlines:

What is unique and also theoretically complex is that the term describes a typical inbetween phenomenon. Atmospheres stand between subjects and objects: one can describe them as object-like emotions, which are randomly cast into a space. But one must at the same time describe them as subjective, insofar as they are nothing without a discerning Subject. But their great value lies exactly in this in-betweeness. (Böhme 2000: 15)

Böhme's description here of the nature of atmospheres in relation to subject and object is compatible, in many respects, to Gibson's definition of affordances:

The meaning or value of a thing is what it affords... What a thing affords a particular observer (or species of observer) points to the organism, the subject. The shape and size and composition and rigidity of a thing, however, point to its physical existence, the object. But these determine what it affords the observer. The affordance points both ways. What a thing is and what it means are not separate, the former being physical and the latter mental, as we are accustomed to believe. (Gibson 1982: 407-8)

In this way Böhme argues that atmospheres can also challenge the separation between an aesthetics of production and one of reception. One can actively create atmospheres, and examine the objects and technology that make them, but it is also 'a matter of reading characteristics as ecstasies, that is, ways in which a thing goes out of itself and modifies the sphere of its surroundings' (Böhme 2000: 15). In a similar vein to Clarke, direct exposure to sound is crucial, 'atmospheres are experienced affectively, and one can only describe their characteristics insofar as one exposes oneself to their presence and experiences them as bodily sensations' (15).

Böhme speaks here of atmospheres specifically in relation to music and acoustic ecology, and considers that music, in its widest sense, has shifted from a historical conception as a product of time - sounds unfolding thematically over a certain duration with an apparent unity that transcends the passing moment - to a more 
contemporary recognition of music's spatial dimension. If the spatial dimension of music has long been a consideration for composers, for Böhme it became a particular point of focus with the emergence of New Music in the $20^{\text {th }}$ century and the development of electronic production and reproduction technologies and techniques. As Böhme elaborates:

The ability to make a sound skitter across the room like an insect or to let it rise above a muffled soundmass and spew sonic fireworks — such things were only made possible by technology, which, in realizing them, directed attention to something which, to a certain extent, has always been part of music's province. (Böhme 2000: 16)

In this enhanced consideration of the spatial, music and sound art can be regarded as the most productive art for the creation of atmospheres:

The discovery that music is the fundamental atmospheric art has solved an old, always annoying and yet inescapable problem of musical theory, i.e. the question: of what does music's so-called emotional effect actually consist? In opposition to the helpless association theories and the theories that called upon fantasy to mediate, the Aesthetics of Atmospheres gives a simple answer to the question: music as such is a modification of space as it is experienced by the body. Music forms and informs the listener's sense of self, (das Sichbefinden) in a space; it reaches directly into his or her corporeal economy. (Böhme 2000: 16)

We can summarise that Böhme's conception of atmospheres situates the listening body as rooted in, and responding to the sculpting of acoustic space, while also acknowledging the producer and the technology that projects sound into this space. Furthermore, it delineates three aspects of the creative use of sound, the producer (projector of atmospheres into space), the listener (a body affected by the particularities of the sound in space) and the sonic material (that which is projected into space and which shapes it and acts upon the listening body), and takes them into consideration simultaneously. Yet, I would argue that while these are part of a whole experience or event, and interact with each other, they are also, in many ways, discrete entities that do their own work. As such they can be brought under separate modes of analysis, either through direct audition or technological means. From this we can deduce that if the atmosphere, the 'in-between', is the site of the experience, 
then neither its comprehension nor its verbal description are without difficulty. As Roland Barthes (1979) suggests, in the discussion of music one is seemingly led inevitably to the adjective and the epithet. While it is tempting to avoid conventional adjectival means to describe mood or emotional quality, this approach does have the advantage of being easily understood. It is also true that a more generalised description of atmosphere avoids the myriad individual connotations that might pertain in relation to any artwork. However, this should not disguise the fact that there are materially quantifiable 'emotive' responses to certain sounds. For example, the sense of anxiety created by low-frequency sounds and 'nonlinear' sounds (Blumstein, Davitian and Kaye 2010) and in more general musical production, at least in Western traditions, certain intervals, key signatures or chords structures are used to create certain 'emotional' effects. So we might suggest that the operation of creative sounds diffused in a specific environment imparts on the listener both a material and an emotional / intellectual influence. What is most important here is the propensity of space to shape and mould the sound diffused within it, and vice versa. So, along with the affective / emotional aspect of atmosphere, there is also the experience and apprehension of the sonics of space as a distinctive signature, a unique marker of place.

\section{THE STRATIFICATION OF SONIC MEDIA}

Given these analytical tools, provided by Gibson and Böhme, how might we mould a method of analysis that will broaden our understanding of both the production and examination of context-based sonic works? Tim Ingold (2007), in his discussion of the soundscape, suggests that there is a fundamental difference between the 'audibility' of an environment and it being made 'aural' through technological reproduction. However, I would emphasise that the difference between experiencing the sound of an environment within that environment and the experience of it played back via a recording is not definitive. In relation to the ecological theory this opens up some very important issues. The fundamental point here is that the same perceptual apparatus is clearly in play for both sets of experiences. That is to say, we have obviously not evolved a new perceptual apparatus in the short amount of time since the invention of audio recording. However, we have become acculturated to the particular sonic qualities of media sources through familiarity: their sounds being an ever-present part of life in technologically developed societies. The ubiquity of sonic media could be seen as pushing the development of a greater degree of auditory 
competences in many listeners and a more analytical mode of listening. Therefore individuals might be considered as becoming increasingly able to distinguish between the specificities of various media sound objects and other sounds stemming from the environment. Yet, the question arises how does an apparatus that evolved to engage with the environment as direct experience engage with its playback in the secondary environment of an exhibition space? ${ }^{\text {ix }}$ In addition, other questions arise in relation to the particularities of the exhibition space itself and the technological means for the diffusion of the work. In response to these questions, and given the exploratory nature of the perceptual apparatus, I would argue, first, that in the exhibition of a sound work the process of audio playback engenders a perceptual and cognitive oscillation between the space of audition and the aural space (re)-presented in the sound work itself. Second, that, a third environment or sphere should also be brought into consideration, namely, the post-production environment. The manipulation and arrangement of sounds in post-production, with regards to panning, binaural positioning and levels of reverberation, are directly working on audio recordings in relation to developing and enhancing spatial effects. ${ }^{\mathrm{x}}$ In short, I would contend that in any sound installation (and even in wider categories of audio work) we perceive three simultaneously occurring strata of acoustic environments: the sound of the space of the recorded environment, the sound of the 'virtual' space of the post-production environment, and the sound of the exhibition space in which the sound work is presented (the work being contoured by the audio speaker array and the acoustics of the space). The listener may perceive these as a unitary audio experience or, depending on the specific address of the work and / or on the particular actions of the listener, as a conscious shifting of attention between or through these different environments. However, for analytical purposes it is useful to identify them and examine their functioning as separate, if integrated, strata or environments. To support this notion of three perceptual spaces, one can observe that most sound artists working with environmental sound will, to a greater or lesser extent, record and / or organise sound in three stages in line with the three strata. That is to say, they will record sounds in the natural / built environment, arrange / modify sounds in post-production (to a greater or lesser extent), and finally diffuse sound through audio speakers and mix sound levels in relation to the acoustics and / or physical parameters of the exhibition space.

With regard to the space of the recorded environment, the initial recording techniques themselves are also predicated on spatial considerations, through the choice of microphones, based on their response patterns, and their placement. These 
recording methods and effects might also be further designed in relation to the diffusion technology (speaker array or headphones), which may have a particular spatial arrangement (stereo, multichannel, surround, binaural etc.). The point of diffusion can be regarded as the interface between the recording and post-production space and the environment of reception. In speaker-based diffusion, the interior or exterior space of exhibition will also operate to further shape and colour the sound.

The delineation of the three audio strata provides a model for understanding the structure of a sound work in relation to the perceptual apparatus. On this foundation, a case-specific analysis enables us to examine a producer's conceptual processes and content generation, alongside audience experience.

\section{CASE STUDY: CHRIS WATSON}

As well as being constructed as installations, context-based sound works also commonly circulate as CD recordings or downloadable files. In light of my emphasis on the mobile perceiving body in relation to ecological perception, it is important to see if the exploratory nature of perception also applies to the 'static' listener when they listen to sound recordings. In addition, are these recordings able to transmit atmospheres and convey the aesthetic and emotional affect of specific environments and places?

My specific interest with Watson's work is that he often endeavours to create what might be described as perceptually coherent sound environments that can be probed and explored by the perceptual apparatus of the listener. However, this broadly 'naturalistic' reproduction should not conceal the constructed nature of the work undertaken to produce his various sound works.

Watson's current practice as a natural history sound recordist for the BBC is predicated on hi-fidelity recording standards and the development of both technical and field-craft techniques needed to meet the demanding remits of this type of work. This, coupled with travel to remote locations, provides Watson with the opportunity to capture sound material of environmental and bioacoustic specificity that would be inaccessible to most. Watson's recorded works or live presentations comprise a number of techniques to create perceptually coherent sonic environments. ${ }^{\mathrm{x}}$ That is to say, we are able to perceive spatial directions, depth and sonic events in a way that is recognisable in relation to our experience of perceiving actual environments. It is Watson's tripartite approach to the layering of sound, both in recording an environment and in the organisation of material in post-production, which is central to 
this effect. Watson creates a foundation layer of the general ambience of an environment, which is used to give a sense of geographical space, then a secondary layer of more detailed sounds of the specific habitat, and then as a top layer, a foregrounded sonic element, such as a species-specific sound or notable environmental sound event. Some of the recording techniques he employs also explore the environment in relation to sound working as a dynamic sonic event. In this instance, the recordings use reflected sound, that is, sound moulded by the topography of the specific place, not just direct recording of its source. Through this layering method, Watson creates a structure of sonic information that affords exploratory perceptual probing across the strata of audio presented in the work.

The spatialisation processes adopted in the post-production environment enhances the spatial qualities already apparent in the natural environments recorded. Watson's drive in Weather Report (2003) to create 'naturalistic' and perceptually coherent audio spaces means that these processes are not always discernable to the listener. That is to say, they maintain, in ecological terms, the invariants in the perceptual register. Having said this, at times, due to the impact of the sounds introduced in terms of volume or specific characteristics, there are instances that make demands on the perceptual processes of the listener and draw attention to the constructed nature of the piece. In later works, such as El Tren Fantasma (2011), the constructed nature of the work is clearly articulated. This is most apparent on the track El Divisadero, where rhythms and resonances and of the train moving through tunnels are extended and looped to create a piece that moves from its basis as field recording to a constructed driving sound piece that is redolent of Watson's earlier 'industrial / electronica' work with the band Cabaret Voltaire.

Another of the techniques Watson uses to construct his work he describes as 'time compression'. For him, time compression involves the selection of significant elements from the durational recording of an environment. In Weather Report Watson foregrounds this technique, as the track listing for the album indicates the degree of 'time compression' used for two of the three tracks: 'Ol-Olool-O, eighteen minute from fourteen continuous hours recorded in Kenya's Masai Mara' and 'The Lapaich, eighteen minutes selected from recordings of a Scottish glen over a four month period of autumn and into winter'. However, this wider selection process is also based upon recording methods and technology chosen to record a sound environment as perceptually coherent as possible. In this way his work affords a perceptual exploration of distant environments and extraordinary spaces, such as the crevices of 
glaciers or the interior of a zebra carcass as vultures are consuming it. While these approaches are not unique to Watson and are used by other artists working with environmental recordings (see Truax 2002, 2012), these highly precise recording and editing techniques form the basis of an aesthetic built on rendering the strangeness and drama of the acoustic world in ways that are also familiar in their structure and spatial organisation.

In Watson's work we can also see more clearly the operation of Böhme's conception of atmospheres. This is most clearly indicated in his albums Outside the Circle of Fire (Touch 1998) and Stepping into the Dark (Touch 1996), which were based on the recording of specific 'atmospheric' places or bioacoustics events. It is worth looking in more detail at Watson's use of the term 'atmospheric'. While not a direct reference to Böhme's usage, it is clearly describing the same area of experience. As Watson states,

the quiet atmosphere of a place, location or habitat can be a revelation - a profound 'presence' made up of the component parts. I tried to capture this real sense of place with my Touch CD - Stepping into the Dark - after experiencing several atmospheres that I felt had a tangible character. (Watson in Toop 2004: 52)

Watson goes on to say that the atmosphere of some of the places he records can fill him with an 'overpowering sense of foreboding'. This can be related to Böhme's broader category of Ecological Nature Aesthetics: the exploration of the relationship between the quality of the environment and human sensibilities and how the environment can be experienced both emotionally and aesthetically. In this regard, Watson's work doesn't simply relate to selecting interesting sound events as objective elements to be recorded in a technically sophisticated manner; it also involves attempting to capture the way sound operates, to use Böhme's terminology, as 'object-like emotions'. In other words, the sounds of place have a tangible sense of emotional intensity that Watson might be able to record and transmit to an audience who has not experienced the place in actuality. This 'atmosphere' might be considered as already apparent, as a product of the multitude of factors that shape the sound as it emanates from and within a specific environment. That is to say, atmospheres, as either 'naturally' occurring or formed through creative practice, are a discernable intensity or event born of particular environmental configurations and, most importantly, they carry meaning. Here, 'sound' can be understood as a dynamic interaction of the sound object, the environment, and the active perceiving body: an event that unfolds in real-time. 
In my Introduction I quoted from Watson's correspondence with David Toop (2004), where Watson sets out a question that can be regarded as a central issue for this paper. To re-cap in brief the statement here, Watson observes that 'the paradox is that some sounds can convey clarity and depth even when played back or broadcast over the compressed mediums ...I'm fascinated by this but can't explain it!' (Watson in Toop 2004: 51). I would argue that, as outlined by the ecological model and my concept of the three strata of audio environments, this 'paradox' can be explained by the ability of the perceptual apparatus to probe and explore sonic space even through the degradation of signal that occurs through the various levels of transmission. That is to say, in spite of this mediation, something of the essential dimensions and atmosphere of the sounds as contoured by a particular environment - its informational structure - remains intact. The compression and noise brought into the signal through processing and transmission does not degrade, at least up to a certain point, the structural integrity and particularities of the sonic information. This is a crucial point of the ecological model: that we consider the perceptual apparatus as engaging with sound (and other sensorial material) as information rather than energy.

It is perhaps the uncompromising search for technical and 'atmospheric' integrity in Watson's approach that makes an examination of his work here so fruitful. That he is consciously exploring the perceptual and emotional elements of environmental sound through intense listening and precise high-resolution recording creates exceedingly productive material to scrutinise in relation to a methodology based on the ecological model of perception.

\section{CONCLUSION}

Gibson's notion of affordances posits that a clear distinction between sound as object and listener as subject is not sustainable, objects being both a function of both matter and mind (Gibson 1982). Gernot Böhme's (2000) concept of atmospheres, which deals with aestheticised sound, similarly challenges the separation of object and subject and formulates an understanding of sound as 'object-like emotions' (Böhme 2000: 15). Given this shared conception, atmospheres might be considered as a means of translating affordances into the area of aesthetics, as both a means of providing perceptual / sensual pleasure and of transmitting knowledge. What is central to the analysis offered here is the way works based on environmental sound, in their specific ways, address the perceptual system through a modulation between creating 
perceptually coherent sound worlds and perceptual challenges and play. In other words, we can see sound work as a shifting mode of address as it oscillates between an attempt at the reconstruction of a sound world that 'fits' with our perception of the everyday environment and work that foregrounds the apparatus of production and presentation and resonant spaces of exhibition. This is a crucial point because it allows the application of the analytical approach I am proposing here to be applied not just to the work of context-based sound artists who attempt to create perceptually coherent sound worlds, such as most of Watson's work examined here, but also to work that moves to a much more 'abstract' mode of address. So it can also incorporate the shift to a more 'musical' mode, as is apparent in Watson's $E l$ Divisadero. Given that many composers making context-based work play with these shifting modalities (e.g. Hildegard Westerkamp; Truax 2012), I suggest that the methodology I propose in this article is applicable to a broader range of work than the case study examined here. To provide one brief example, Janet Cardiff's headphone based 'audiowalk' The Missing Voice (Case Study B) (1999) uses binaural recording methods and a mix of pre-recorded sounds encountered on the designated route (in this work, the backstreets of Whitechapel, London), various narrative voices and additional found sound effects, all of which blend and interact with the sounds bleeding in from the environment as the listener completes the walk. Missing Voice (Case Study B) therefore makes continued demands on the listener to perceptually probe and unravel the three strata of audio environments: the sound of the space of the recorded environment, the sound of the 'virtual' space of the post-production environment, and the sound of the exhibition space in which the sound work is presented.

Böhme's notion of atmospheres in relation to creative sound production also needs to be considered with reference to his broader category of 'Ecological Nature Aesthetics': the exploration of the relationship between the quality of the environment and human sensibilities and how the environment can be experienced aesthetically. I would suggest that the use of the term 'nature' should clearly not preclude the possibility of experiencing urban environments 'aesthetically'. These concepts of Ecological Nature Aesthetics and atmospheres underline a consideration of the context-specific on an affective level. That is to say, what sound-based work affords is an engagement with atmosphere as a form of sensory experience or knowledge: embodied pleasures that might open up other understandings.

If Eric Clarke made a valuable contribution in applying the ecological theory of perception to music, then contemporary sound art practice, given the range of 
technological methods for diffusion, offers even more possibilities to work with the grain of the perceptual system's exploratory nature. I would go further and claim that there is a clear distinction to be made in sound-based artworks between aesthetic strategies built on the figure of the static perceiver - the 'perceptual management' of listeners around an ideal position, the 'sweet spot' - and sound work that structures its aesthetic on the potential of audio technologies to address the 'mobile perceiver', as described by Gibson within the ecological model. Where I believe the methodology outlined in this paper is of particular, additional value is in the way it can be deployed to examine the functions of both producer and receiver. This is not to return to an older model within communications or media theory of the 'encoder / decoder', but, rather, to advance a methodology based on the consideration of a shared perceptual apparatus in play throughout the process, informing both production modes and listener experience.

\section{REFERENCES}

Augé, Marc 1995. Non-Places: Introduction to an Anthropology of Supermodernity. London: Verso.

Barthes, R. 1979. The Grain of The Voice. In S. Heath (ed. \& trans.) Image, Music, Text, $2^{\text {nd }}$ edition. London: Fontana, 179-89.

Blumstein, T. D., Davitian, R. and Kaye, P. D. 2010. Do Film Soundtracks Contain Nonlinear Analogues to Influence Emotion? Biology Letters 6(6): 751-4.

Böhme, G. 2000. Acoustic Atmospheres: A Contribution to the Study of Ecological Aesthetics. Soundscape: The Journal of Acoustic Ecology 1(1): 14-19.

Clarke, E. 2005. Ways of Listening: An Ecological Approach to the Perception of Musical Meaning. Oxford: Oxford University Press.

Cox, C. 2011. Beyond Representation and Signification: Toward a Sonic Materialism. Journal of Visual Culture 10(2): 145-61.

Crary, J. 1999. Suspensions of Perception: Attention, Spectacle, and Modern Culture. Cambridge, MA: MIT Press.

Gibson, J.J. 1966. The Senses Considered as Perceptual Systems.

Boston: Houghton Mifflin.

Gibson, J.J. 1979. The Ecological Approach to Visual Perception. Boston: Houghton Mifflin. 
Gibson, J.J. 1982. Notes on Affordances. In E. Reed and R. Jones (eds.) Reasons for Realism: Selected Essays of James J. Gibson. Hillsdale, NJ: Lawrence Erlbaum Associates, 401-18.

Hecker, F. 2009. Sound Out of Line: In Conversation with Florian Hecker: Urbonomic. https://www.urbanomic.com/wp-content/uploads/2015/06/Urbanomic_Document_UFD003.pdf

Ingold, T. 2007. Against Soundscape. In A. Carlyle (ed.) Autumn Leaves: Sounds and the Environment in Artistic Practice. Paris: Double Entendre, 10-13.

Katz, B. 2014. Mastering Audio. The Art and The Science, 3rd edition. Oxford: Focal Press.

Kwon, M. 2004. One Place After Another: Site-Specific Art and Locational Identity. Cambridge, MA: MIT Press.

Risset, J. C. 1996. Real-World Sounds and Simulacra in my Computer Music. Contemporary Music Review 15(1): 29-47.

Schafer, R. M. 1977. The Tuning of the World. New York: Knopf; reprinted as The Soundscape: Our Sonic Environment and The Tuning of the World. Rochester, VT: Destiny Books, 1994.

Sterne, J. 2006. The mp3 as Cultural Object. New Media and Society 8(15): 825-42.

Toop, D. 2004. Haunted Weather. London: Serpent's Tail.

Truax, B. 2002. Genres and Techniques of Soundscape Composition as Developed at Simon Fraser University. Organised Sound 7(1): 5-14.

Truax, B. 2012. Sound, Listening and Place: The Aesthetic Dilemma. Organised Sound 17(3): 193-201.

\section{DISCOGRAPHY}

Cardiff, J. (1999) The Missing Voice (Case Study B) [Installation / Audiowalk], Whitechapel Gallery and environs: https://www.artangel.org.uk/project/the-missingvoice-case-study-b/

Watson, C. 2011. El Tren Fantasma [CD]. London: Touch Music, TO:42-CD

Watson, C. 2003. Weather Report [CD]. London: Touch Music, TO:47-CD

Watson, C. 1998. Outside the Circle of Fire [CD]. London: Touch Music, TO:37-CD

Watson, C. 1996. Stepping into the Dark [CD]. London: Touch Music, TO:27-CD

\section{Endnotes}


${ }^{i}$ For the purposes of this article context-based sound work includes site-specific installations and environmental recordings, which might also be described as field recordings, phonography, soundscape composition or acoustic ecology research.

ii One might consider the pioneering work of R. Murray Schafer (1977), which has been such an important influence on much context-based composition, as motivated by an awareness of the advancing formation of sonic 'non-space' and the need to understand and challenge this process.

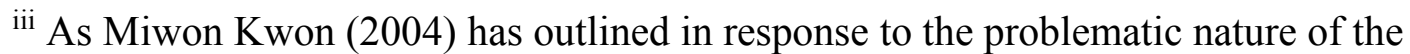
term 'site-specific' and some of the works associated with it, other terms such as 'siteresponsive', 'context-specific', 'debate-specific', 'audience-specific', 'communityspecific' and 'project-based' emerged to more clearly describe the various approaches to the idea of the 'site'.

iv The first major exhibition of sound art in Britain, 'Sonic Boom - The Art of Sound' (Hayward Gallery, South Bank Centre London 27. April -18. June 2000) clearly indicates how a large number of what might be considered 'sound' works also involve moving image or three dimensional media as components of the work. This is particularly true if we also consider speakers as sculptural objects in their own right.

' Jonathan Sterne's (2006) work on the mp3 file format suggests that the success of the mp3's compression codec is due to its use of 'perceptual coding': the discarding of frequencies that are not regarded as necessary for audition by the average listener. This is coupled to the question of what is 'acceptable' quality for the music consumer, given the largely casual forms of listening (via iPods, smart phones, laptops and so on) that are a feature of the mp3's success as an 'artifact' for the distribution and consumption of music. While Sterne's commentary helps suggest some answers to the 'clarity' aspect of Watson's observations, it does not deal with the issue of 'depth'.

vi This aspect is covered by Gibson's important concept of 'affordance'. While 'affordance' works as a model for all advanced animals, it is assumed that different perceptual faculties are in operation in different species.

${ }^{\text {vii }}$ For example, the ecological theory has been influential on Jean-Claude Risset (1996) in relation to his work on sound synthesis and more recently in the installation soundwork of Florian Hecker (2009), who even named a composition Affordance (2013).

viii One interesting example of this more inclusive approach is that of Enactive Cognition (Varela, F. et al.1991), which views cognition as embodied action and occurs through directed interaction between the mind / body and the environment. Varela's conception of enactive cognition draws on the phenomenology of MerleauPonty and Buddhist thought, and closely links cognition to evolutionary processes.

ix In this context, 'exhibition space' refers to the point of consumption of the work, albeit site, gallery, concert hall or home via speaker arrays or headphones.

${ }^{x}$ Many of these considerations and techniques are of course generally applied in the mastering of music recordings as well as in sound art work (see Katz 2014).

${ }^{x i}$ My descriptions of Chris Watson's techniques have been gleaned from attending various talks given by him rather than from published sources. 